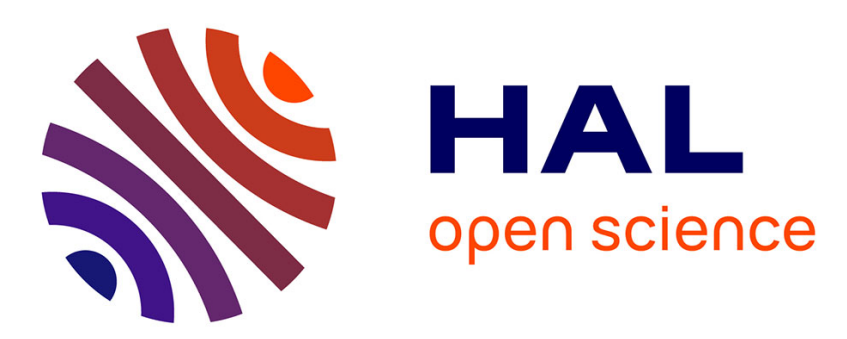

\title{
Composite Nonlinear Feedback-based Bounded Formation Control of Multi-quadrotor Systems
}

\author{
Zhicheng Hou, Isabelle Fantoni
}

\section{To cite this version:}

Zhicheng Hou, Isabelle Fantoni. Composite Nonlinear Feedback-based Bounded Formation Control of Multi-quadrotor Systems. European Control Conference (ECC 2016), Jun 2016, Aalborg, Denmark. hal-01306352

\section{HAL Id: hal-01306352 \\ https://hal.science/hal-01306352}

Submitted on 22 Apr 2016

HAL is a multi-disciplinary open access archive for the deposit and dissemination of scientific research documents, whether they are published or not. The documents may come from teaching and research institutions in France or abroad, or from public or private research centers.
L'archive ouverte pluridisciplinaire HAL, est destinée au dépôt et à la diffusion de documents scientifiques de niveau recherche, publiés ou non, émanant des établissements d'enseignement et de recherche français ou étrangers, des laboratoires publics ou privés. 


\title{
Composite Nonlinear Feedback-based Bounded Formation Control of Multi-quadrotor Systems
}

\author{
Zhicheng HOU and Isabelle FANTONI
}

\begin{abstract}
This paper presents a bounded formation controller for multiple quadrotors (UAVs) system with leaderfollower structure. Considering the actuator saturation of the quadrotors, the hyperbolic function is used in the formation controller design. The composite nonlinear feedback is integrated in the formation controller in order to improve the formation performance. The simulation and experimental results show that the formation task is achieved with small overshoot and rapid response speed.
\end{abstract}

\section{INTRODUCTION}

The cooperative control of multi-quadrotor systems has recently attracted the attention. The manipulation and transportation of large payloads by using multiple UAVs are investigated in [1] and [2]. The problem of cooperative surveillance in large outdoor areas by a fleet of micro aerial vehicles is studied in [3]. The applications such as search and rescue are covered in [4].

In practice, the inputs of a quadrotor are subject to actuator saturations. This problem is taken into account in the quadrotor controller design in the literature. For instance, a nested saturation controller is proposed in [5]. An algorithm of feasible trajectories planning is investigated in [6], where the input constraints of quadrotor are considered.

To the best of our knowledge, the input saturations are not very much considered in the multi-quadrotor systems control. In [7], a low gain feedback control is proposed to avoid the saturation of the system, such that a semi-global leader-follower consensus of linear multi-agent system is achieved. In the formation control of multi-UAV systems, a large formation error can generate a large control output. This could lead to big attitude angles for the UAVs of the formation. Although the low gain controller [7] can guarantee the control output small, the response speed of the closedloop system is low.

In this paper, the formation of quadrotors with LeaderFollower (L-F) structure is investigated. Considering the input saturation of the quadrotor, a bounded formation controller is developed. The formation control strategy is proposed aiming at investigating a bounded differentiable formation controller and obtaining the closed-loop system with fast response speed and small overshoot.

\footnotetext{
Zhicheng HOU and Isabelle FANTONI are with Sorbonne universités, Université de technologie de Compiègne, CNRS, UMR 7253 Heudiasyc, 60200 Compiègne, France. zhicheng.hou/isabelle.fantoniahds.utc.fr

This work was carried out and funded in the framework of the Labex MS2T (Reference ANR-11-IDEX-0004-02) and the ROBOTEX Equipment of Excellence (Reference ANR-10-EQPX-44). They were supported by the French Government, through the program Investments for the future managed by the National Agency for Research.
}

In recent years, authors in [8] [9] have proposed and implemented a "composite nonlinear feedback" (CNF) control to improve the performance of a system. The objective of this control method is to reduce the overshoot and meanwhile, keep a rapid rising response.

In this paper, we are inspired by the CNF control method to improve the performance of the formation of UAVs. Considering the input saturation of the quadrotors, a hyperbolic tangent function is used instead of a standard saturated function, which has two non-differentiable points when the function gets saturated. The high-order derivatives of the formation controller of each UAV are needed for the attitude control. Therefore, if we use the standard saturated function, an infinite derivative will be generated at the nondifferentiable points. In contrary, the hyperbolic tangent function is always differentiable and has bounded high-order derivatives.

The paper is organized as follows. The modeling and control of one quadrotor are presented in section II. The formation control design and the stability analysis are shown in section III. Simulation and experimental results are given in section IV. Finally, conclusions are stated in section V.

\section{Modeling AND CONTROL OF ONE UAV}

\section{A. Modeling}

The dynamics of a quadrotor is modeled as the motion of a rigid body in 3-D space under a thrust force and three moments, which are generated by the thrust forces of the four rotors. The orientation of the quadrotor with respect to the inertial frame is represented by the rotation matrix $R_{i} \in$ $\mathrm{SO}(3)$, where $\mathrm{SO}(3)$ represents a special orthogonal group, and whose determinant is one.

Vector $\mathcal{X}_{i}=\left[X_{i}, Y_{i}, Z_{i}\right]^{T}$ represents the coordinates of the center of mass of a quadrotor in the fixed inertial frame $o_{e} x_{e} y_{e} z_{e}$. The Euler angles (roll, pitch and yaw) are represented by the vector $\Theta_{i}=\left[\phi_{i}, \theta_{i}, \psi_{i}\right]^{T}$. The inertia matrix $J=\operatorname{diag}\left\{I_{x_{b}}, I_{y_{b}}, I_{z_{b}}\right\}$ of a quadrotor is diagonal, if $I_{x_{b}}, I_{y_{b}}$ and $I_{z_{b}}$ represent the moments of inertia with respect to the bodyfixed frame $x_{b}, y_{b}$ and $z_{b}$ respectively. The angular velocity of the quadrotor $i$ in the body-fixed frame is represented by $\Omega_{i} \in \mathbb{R}^{3}$. The function $S(\cdot): \mathbb{R}^{3} \rightarrow \mathbb{R}^{3 \times 3}$ represents an operation that transforms a vector in $\mathbb{R}^{3}$ to a skew-symmetric matrix $\mathbb{R}^{3 \times 3}$.

The roll, pitch and yaw moments are represented by $\tau_{i}=\left[\tau_{\phi_{i}}, \tau_{\theta_{i}}, \tau_{\psi_{i}}\right]^{T} \in \mathbb{R}^{3}$. The thrust force is represented by $F_{T_{i}}$. The rotation matrix $R_{i}$ from the body-fixed frame to the inertial frame is with the sequence of roll-pitch-yaw, thus, 
we obtain the translational dynamics as follows

$$
\begin{aligned}
& \ddot{X}_{i}=\left(\sin \psi_{i} \sin \phi_{i}+\cos \psi_{i} \cos \phi_{i} \sin \theta_{i}\right) \frac{F_{T_{i}}}{m} \\
& \ddot{Y}_{i}=\left(\cos \phi_{i} \sin \psi_{i} \sin \theta_{i}-\cos \psi_{i} \sin \phi_{i}\right) \frac{F_{T_{i}}}{m} \\
& \ddot{Z}_{i}=-g+\left(\cos \theta_{i} \cos \phi_{i}\right) \frac{F_{T_{i}}}{m}
\end{aligned}
$$

where $m$ represents the mass of a quadrotor and $g$ represents the gravity.

Let us denote

$$
T_{i}=\left[\begin{array}{ccc}
1 & 0 & -\sin \theta_{i} \\
0 & \cos \phi_{i} & \cos \theta_{i} \sin \phi_{i} \\
0 & -\sin \phi_{i} & \cos \theta_{i} \cos \phi_{i}
\end{array}\right]
$$

and

$$
\tilde{T}_{i}=\left[\begin{array}{ccc}
\tan \theta_{i} & 0 & \cos \theta_{i}+\tan \theta_{i} \sin \theta_{i} \\
0 & -\cos \theta_{i} & 0 \\
\sec \theta_{i} & 0 & \tan \theta_{i}
\end{array}\right]
$$

We can rewrite the rotational dynamics as follows

$$
\begin{aligned}
{\left[\begin{array}{c}
\ddot{\phi}_{i} \\
\ddot{\theta}_{i} \\
\ddot{\psi}_{i}
\end{array}\right]=\left(J T_{i}\right)^{-1} \tau_{i}+\tilde{T}_{i} \cdot\left[\begin{array}{c}
\dot{\phi}_{i} \dot{\theta}_{i} \\
\dot{\phi}_{i} \dot{\psi}_{i} \\
\dot{\theta}_{i} \dot{\psi}_{i}
\end{array}\right] } \\
+\left(J T_{i}\right)^{-1} S^{T}\left(T_{i} \cdot\left[\begin{array}{c}
\dot{\phi}_{i} \\
\dot{\theta}_{i} \\
\dot{\psi}_{i}
\end{array}\right]\right) J T_{i} \cdot\left[\begin{array}{c}
\dot{\phi}_{i} \\
\dot{\theta}_{i} \\
\dot{\psi}_{i}
\end{array}\right]
\end{aligned}
$$

The dynamics of a quadrotor can be generally divided into two parts, namely, the translational dynamics (1) in outer-loop, and the rotational dynamics (2) in inner-loop respectively.

\section{B. Attitude control}

Since the attitude angles and the angular velocities are measurable, the torque vector $\tau_{i}$ is given as follows

$$
\tau_{i}=J T_{i} \bar{\tau}_{i}-J T_{i} \tilde{T}_{i}\left[\begin{array}{c}
\dot{\phi}_{i} \dot{\theta}_{i} \\
\dot{\phi}_{i} \dot{\psi}_{i} \\
\dot{\theta}_{i} \dot{\psi}_{i}
\end{array}\right]-S^{T}\left(T_{i} \cdot \dot{\Theta}_{i}\right) J T_{i} \cdot \dot{\Theta}_{i}
$$

where

$$
\bar{\tau}_{i}=\ddot{\Theta}_{i}^{d}-\frac{k_{2 \Theta_{i}}}{\varepsilon}\left(\dot{\Theta}_{i}-\dot{\Theta}_{i}^{d}\right)-\frac{k_{1 \Theta_{i}}}{\varepsilon^{2}}\left(\Theta_{i}-\Theta_{i}^{d}\right)
$$

Notations $k_{1 \Theta_{i}}$ and $k_{2 \Theta_{i}}$ represent two diagonal positivedefinite gain matrices. Notations $\Theta_{i}^{d}$, $\dot{\Theta}_{i}^{d}$ and $\ddot{\Theta}_{i}{ }^{d}$ represent the desired Euler angles vector and their derivatives. The scalar $\varepsilon \in(0,1]$.

Substituting (3) into (2), we obtain

$$
\ddot{\Theta}_{i}=\ddot{\Theta}_{i}^{d}-\frac{k_{2 \Theta_{i}}}{\varepsilon}\left(\dot{\Theta}_{i}-\dot{\Theta}_{i}^{d}\right)-\frac{k_{1 \Theta_{i}}}{\varepsilon^{2}}\left(\Theta_{i}-\Theta_{i}^{d}\right)
$$

Then, the attitude angles in $\Theta_{i}$ can track $\Theta_{i}^{d}$ exponentially. The converging speed is decided by the gain matrices $k_{1 \Theta_{i}}$ and $k_{2 \Theta_{i}}$. Let us denote $e_{\Theta_{i}}=\Theta_{i}-\Theta_{i}^{d}$ and $\tilde{e}_{\Theta_{i}}=\varepsilon e_{\Theta_{i}}$, then, we can rewrite (5) as follows

$$
\varepsilon \frac{d}{d t}\left[\begin{array}{c}
e_{\Theta_{i}} \\
\dot{\tilde{e}}_{\Theta_{i}}
\end{array}\right]=\left[\begin{array}{cc}
0_{3 \times 3} & I_{3} \\
-k_{1 \Theta_{i}} & -k_{2 \Theta_{i}}
\end{array}\right]\left[\begin{array}{c}
e_{\Theta_{i}} \\
\dot{\tilde{e}}_{\Theta_{i}}
\end{array}\right]
$$

If we define $d \bar{t} / d t=1 / \varepsilon$, then, we have

$$
\frac{d\left[\begin{array}{ll}
\varepsilon e_{\Theta_{i}} & \varepsilon \dot{\tilde{e}}_{\Theta_{i}}
\end{array}\right]^{T}}{d t}=\frac{d\left[\begin{array}{ll}
e_{\Theta_{i}} & \dot{\tilde{e}}_{\Theta_{i}}
\end{array}\right]^{T}}{d \bar{t}}
$$

such that the dynamics of the attitude errors in (6) can be represented in the time scale $\bar{t}$ as follows.

$$
\frac{d\left[\begin{array}{ll}
e_{\Theta_{i}}(\bar{t}) & \dot{\tilde{e}}_{\Theta_{i}}(\bar{t})
\end{array}\right]^{T}}{d \bar{t}}=\left[\begin{array}{cc}
\boldsymbol{0}_{3 \times 3} & I_{3} \\
-k_{1 \Theta_{i}} & -k_{2 \Theta_{i}}
\end{array}\right]\left[\begin{array}{c}
e_{\Theta_{i}}(\bar{t}) \\
\dot{\tilde{e}}_{\Theta_{i}}(\bar{t})
\end{array}\right]
$$

It is not difficult to verify that the origin of (7) is exponentially stable, such that we obtain

$$
\left[\begin{array}{c}
e_{\Theta_{i}}(\bar{t}) \\
\dot{\tilde{e}} \Theta_{i}(\bar{t})
\end{array}\right]=\exp \left(\left[\begin{array}{cc}
\boldsymbol{O}_{3 \times 3} & I_{3} \\
-k_{1 \Theta_{i}} & -k_{2 \Theta_{i}}
\end{array}\right] \bar{t}\right)\left[\begin{array}{c}
e_{\Theta_{i}}\left(\bar{t}_{0}\right) \\
\dot{\tilde{e}}_{\Theta_{i}}\left(\bar{t}_{0}\right)
\end{array}\right]
$$

The initial value $\bar{t}_{0}=0$ is defined at $t=t_{k}$, where $t_{k}$ is an arbitrary time instant in time scale $t$. Then the new time variable satisfies $\bar{t}=\left(t-t_{k}\right) / \varepsilon$. Then we obtain $t=t_{k}+\varepsilon \bar{t}$. If $\varepsilon$ is sufficiently small, the variable $t$ slowly varies in time scale $\bar{t}$. Intuitively, the time variable $t$ is not "sensitive" to the change of $\bar{t}$ because of the small $\varepsilon$. Since $\left[\begin{array}{cc}\boldsymbol{0}_{3 \times 3} & I_{3} \\ -k_{1 \Theta_{i}} & -k_{2 \Theta_{i}}\end{array}\right]$ is negative definite, there exists a finite time $\bar{t}_{a}$ in time scale $\bar{t}$ such that $\exp \left(\left[\begin{array}{cc}\boldsymbol{0}_{3 \times 3} & I_{3} \\ -k_{1 \Theta_{i}} & -k_{2 \Theta_{i}}\end{array}\right] \bar{t}\right)$ converges to a small neighborhood $\Omega\left(e_{\Theta_{i}}, \dot{\tilde{e}}_{\Theta_{i}}\right)$ of the equilibrium point $\left[\begin{array}{ll}e_{\Theta_{i}} & \dot{\tilde{e}}_{\Theta_{i}}\end{array}\right]=\boldsymbol{0}_{6}$. This convergence dose not depend on the time instance $t_{k}$ and the translational states $\mathcal{X}_{i}\left(t_{k}\right)$. Thus, the equilibrium point of system (7) is exponentially stable, uniformly in $\left(t, X_{i}(t), Y_{i}(t)\right)$.

We recall that the torques in $\tau_{i}$ and the total thrust force $F_{T_{i}}$ are generated by the thrusts of the four propellers of the quadrotor. In practice, the propellers have limit power.

According to (5), in order to prevent $\tau_{i}$ from hitting the input saturation, the terms $\Theta_{i}^{d}, \dot{\Theta}_{i}^{d}$ and $\ddot{\Theta}_{i}^{d}$ should be bounded. Additionally, we require that the absolute values of the desired pitch and roll angles are smaller than $\pi / 2$.

\section{Altitude control}

In this work, the desired altitude $Z_{i}^{d}$ of each UAV is constant. In other words, the formation takes place in $o_{e} x_{e} y_{e}$ plan. The thrust force is given as follows

$$
F_{T_{i}}=\frac{m g+m \cdot u_{i}^{Z}}{\cos \theta_{i} \cos \phi_{i}}
$$

where $u_{i}^{Z}$ represents the altitude controller.

Substituting $F_{T_{i}}$ in (1), we obtain

$$
\begin{aligned}
& \ddot{X}_{i}=\left(\sin \psi_{i} \frac{\tan \phi_{i}}{\cos \theta_{i}}+\cos \psi_{i} \tan \theta_{i}\right)\left(u_{i}^{Z}+g\right) \\
& \ddot{Y}_{i}=\left(\sin \psi_{i} \tan \theta_{i}-\cos \psi_{i} \frac{\tan \phi_{i}}{\cos \theta_{i}}\right)\left(u_{i}^{Z}+g\right) \\
& \ddot{Z}_{i}=u_{i}^{Z}
\end{aligned}
$$

We observe from (10) that the altitude control is decoupled with the planar translational dynamics $\left(X_{i}\right.$ and $\left.Y_{i}\right)$. Thus, we give the altitude controller as follows

$$
u_{i}^{Z}=\sigma_{b}\left(-k_{2 Z} \dot{Z}_{i}-k_{1 Z}\left(Z_{i}-Z_{i}^{d}\right)\right)
$$


Function $\sigma_{b}(\cdot)$ represents a standard saturation function.

$$
\sigma_{b}(a)=\operatorname{sgn}(a) \min \{b,|a|\}
$$

where "sgn" represents the sign function. We select $b=1$. The controller (11) asymptotically stabilizes the altitude.

Since the altitude control is decoupled with the planar translational dynamics, the convergence of $Z_{i}$ do not depend on the other states of the quadrotors except $Z_{i}$ and $\dot{Z}_{i}$. When the altitude of the quadrotors gets stabilized, $u_{i}^{Z} \approx 0$.

\section{Formation CONTROLLER DESIGN}

Since the planar translational motions are generated by the attitude angles, the formation controller is to obtain the proper desired attitude angles $\Theta_{i}^{d}$. If we design the desired attitude angles as follows

$$
\begin{aligned}
\theta_{i}^{d} & =\arctan \left(\frac{u_{i}^{X}}{g}\right) \\
\phi_{i}^{d} & =\arctan \left(\frac{-u_{i}^{Y} \cos \left(\arctan \left(u_{i}^{X} / g\right)\right)}{g}\right) \\
\psi_{i}^{d} & =0
\end{aligned}
$$

where $u_{i}^{X}$ and $u_{i}^{Y}$ represent the formation controllers in direction $x_{e}$ and $y_{e}$ in the inertial frame. Then, the planar translational dynamics of UAV $i$ yields

$$
\begin{aligned}
\ddot{X}_{i} & =u_{i}^{X}+\delta_{i}^{X} \\
\ddot{Y}_{i} & =u_{i}^{Y}+\delta_{i}^{Y}
\end{aligned}
$$

The terms $\delta_{i}^{X}$ and $\delta_{i}^{Y}$ have the following expression.

$$
\begin{aligned}
\delta_{i}^{X}=\left(g+u_{i}^{Z}\right) \cdot & \left(\sin \Delta \psi_{i} \cdot \frac{\tan \left(\phi_{i}^{d}+\Delta \phi_{i}\right)}{\cos \left(\theta_{i}^{d}+\Delta \theta_{i}\right)}+\right. \\
& \left.\cos \Delta \psi_{i} \cdot \tan \left(\theta_{i}^{d}+\Delta \theta_{i}\right)-\frac{g}{g+u_{i}^{Z}} \tan \theta_{i}^{d}\right) \\
\delta_{i}^{Y}=\left(g+u_{i}^{Z}\right) \cdot & \left(-\cos \Delta \psi_{i} \cdot \frac{\tan \left(\phi_{i}^{d}+\Delta \phi_{i}\right)}{\cos \left(\theta_{i}^{d}+\Delta \theta_{i}\right)}+\right. \\
& \left.\sin \Delta \psi_{i} \cdot \tan \left(\theta_{i}^{d}+\Delta \theta_{i}\right)+\frac{g}{g+u_{i}^{Z}} \frac{\tan \phi_{i}^{d}}{\cos \theta_{i}^{d}}\right)
\end{aligned}
$$

where $\Delta \phi_{i}, \Delta \theta_{i}$ and $\Delta \psi_{i}$ represent the tracking error of the attitude angles $\Theta_{i}-\Theta_{i}^{d}=\left[\Delta \phi_{i}, \Delta \theta_{i}, \Delta \psi_{i}\right]$. We can observe that $\delta_{i}^{X}$ and $\delta_{i}^{Y}$ are in terms of $u_{i}^{Z}$, and $\Theta_{i}-\Theta_{i}^{d}$. According to (8), the attitude tracking error $\Theta_{i}-\Theta_{i}^{d}$ is bounded (since $\Theta_{i}^{d}$ is bounded) and exponentially converges to the origin. Additionally, $\left|u_{i}^{Z}\right| \leq 1$ and $u_{i}^{Z} \rightarrow 0$ when the altitude is stabilized. Thus, we obtain that $\delta_{i}^{X}$ and $\delta_{i}^{Y}$ are bounded.

\section{A. Leader-Follower formation of UAVs}

In this paper, an L-F formation is considered. The indices of the UAVs compose a set $\mathcal{V}=\{1,2, \ldots, n\}$, where $n$ is the number of the UAVs in the formation. The subset $\mathcal{V}_{L} \subseteq$ $\mathcal{V}$ represents the indices of the leaders, therefore, $\mathcal{V}-\mathcal{V}_{L}$ represents the set of followers.

Firstly, we define the "formation task" for a multiquadrotor system as follows.

Definition 1 (Formation task): A formation task for a multi-quadrotor system with L-F architecture is represented by a desired formation trajectory (given to the leader(s)) and a desired geometric pattern (desired inter-distance and orientation between neighboring quadrotors) for the group of quadrotors.

The formation task describes the desired integral behaviors of the multiple quadrotors. The objective of the formation control is to accomplish the formation task, i.e., the quadrotors keep the desired pattern and track a given trajectory by using the formation controllers.

We consider the planar formation of UAVs. The desired altitude is given constant. We denote by $x_{i}=\left[X_{i}, Y_{i}\right]^{T}$ and $\dot{x}_{i}=\left[\dot{X}_{i}, \dot{Y}_{i}\right]^{T}$ the planar position and velocity vectors for UAV $i$. Each UAV can obtain the relative positions and velocities with respect to its neighbors, which can be represented by vectors $\left[\left(x_{i}-x_{j}\right)^{T},\left(\dot{x}_{i}-\dot{x}_{j}\right)^{T}\right]^{T}, j \in \mathcal{N}_{i}$. If the UAV is a leader, besides the foregoing measurements, it can also obtain the relative position and velocity with respect to the formation trajectory $\left[\left(x_{i}-r(t)\right)^{T},\left(\dot{x}_{i}-\dot{r}(t)\right)^{T}\right]^{T}$, where we denote by $r(t)=\left[r_{X}(t), r_{Y}(t)\right]^{T}$ the reference formation trajectory (RFT).

The rigid formation of quadrotors are considered in this paper. Thus, the formation controller is designed to guarantee that all the quadrotors track the RFT with some constant biases $d_{i 0}=\left[d_{i 0}^{X}, d_{i 0}^{Y}\right] \in \mathbb{R}^{2}$, such that the quadrotors keep a constant formation pattern. Therefore, the desired trajectory for each UAV should satisfy $x_{i}^{d}(t)-r(t)=d_{i 0}$ and $\dot{x}_{i}^{d}(t)-$ $\dot{r}(t)=0$, then, we obtain

$$
x_{i}^{d}(t)=d_{i 0}+r(t) \text { and } \dot{x}_{i}^{d}(t)=\dot{r}(t)
$$

An example of four UAVs with rigid formation task is shown in Fig.1.

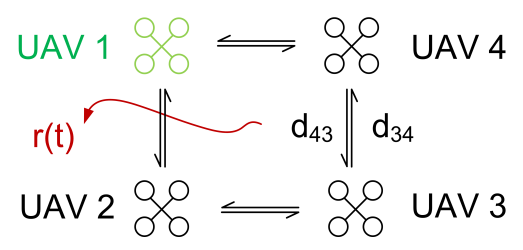

Fig. 1. Rigid formation task for four UAVs, where UAV 1 is a leader. The objective is to track the trajectory $r(t)$ with biases $d_{10}=[-1,1]^{T}, d_{20}=$ $[-1,-1]^{T}, d_{30}=[1,-1]^{T}$ and $d_{40}=[1,1]^{T}$.

However, the RFT $r(t)$ is not available for the followers, therefore, the desired trajectory $x_{i}^{d}(t)$ for UAV $i$ is not explicitly available. The behavior of each UAV depends on its neighbors states (and $r(t)$, if it is a leader), then, the formation task is finally achieved. The bias with respect to the RFT is attained implicitly by the constant inter-distances between a UAV and its neighbors. Then, the formation problem becomes: how to find the available desired trajectory for UAV $i \in \mathcal{V}$.

Let us make a sum of the relative position state vectors. Note that we drop the explicit expression of time in the related terms for the sake of simplicity.

$$
\begin{array}{cc}
\sum_{j \in \mathcal{N}_{i}}\left(x_{i}-x_{j}-d_{i j}\right) & \text { if } i \in \mathcal{V}-\mathcal{V}_{L} \\
\sum_{j \in \mathcal{N}_{i}}\left(x_{i}-x_{j}-d_{i j}\right)+x_{i}-r-d_{i 0} & \text { if } i \in \mathcal{V}_{L}
\end{array}
$$


The inter-distance is given by $d_{i j}=d_{i 0}-d_{j 0}$. Then, equations (15) can be rewritten as follows

$$
\begin{array}{cc}
\sum_{j \in \mathcal{N}_{i}}\left(x_{i}-r-d_{i 0}-\left(x_{j}-r-d_{j 0}\right)\right) & \text { if } i \in \mathcal{V}-\mathcal{V}_{L} \\
\sum_{j \in \mathcal{N}_{i}}\left(x_{i}-r-d_{i 0}-\left(x_{j}-r-d_{j 0}\right)\right)+x_{i}-r-d_{i 0} & \text { if } i \in \mathcal{V}_{L}
\end{array}
$$

We introduce the available desired trajectory for each UAV as follows

$$
\bar{x}_{i}^{d}=\left\{\begin{array}{cc}
\frac{1}{\left|\mathcal{N}_{i}\right|} \sum_{j \in \mathcal{N}_{i}}\left(x_{j}+d_{i j}\right) & \text { if } i \in \mathcal{V}-\mathcal{V}_{L} \\
\frac{1}{\left|\mathcal{N}_{i}+1\right|}\left(\sum_{j \in \mathcal{N}_{i}}\left(x_{j}+d_{i j}\right)+r+d_{i 0}\right) & \text { if } i \in \mathcal{V}_{L}
\end{array}\right.
$$

we then observe that $\bar{x}_{i}^{d}$ is available for UAV $i$. We rewrite equation (15) in matrix form for all the quadrotors as follows

$$
\left[\begin{array}{c}
x_{1}-\bar{x}_{1}^{d} \\
\vdots \\
x_{n}-\bar{x}_{n}^{d}
\end{array}\right]=\left(G \otimes I_{2}\right)\left[\begin{array}{c}
x_{1}-x_{1}^{d} \\
\vdots \\
x_{n}-x_{n}^{d}
\end{array}\right]
$$

where $G \in \mathbb{R}^{n \times n}$ represents the normalized interaction matrix (see [10]). The symbol $\otimes$ represents the Kronecker product. The formation task is achieved if $G$ is invertible and for each UAV $i \in \mathcal{V}, x_{i}-\bar{x}_{i}^{d} \rightarrow 0$ asymptotically. According to [10], $G$ is invertible if the graph of the multi-UAV system is connected and at least one leader exists. In this paper, we assume that this condition is satisfied. In the following subsection, we will investigate the formation controller which stabilizes the formation error $x_{i}-\bar{x}_{i}^{d} \rightarrow 0$.

\section{B. Formation control}

We firstly consider the nominal model of (14) by setting $\delta_{i}^{X}$ and $\delta_{i}^{Y}$ null. The dynamics of $X_{i}$ and $Y_{i}$ are similar, thus, we consider the dynamics of $X_{i}$ for example. Note that in the sequel, the superscript " $X$ " is omitted for the sake of simplicity. Then, we have

$$
\ddot{X}_{i}=u_{i}
$$

Let us denote by $e_{i X}=X_{i}-\bar{X}_{i}^{d}$ the formation error in $x_{e}$ direction. Let us define a nonlinear function $\rho_{i}: \mathbb{R}^{2} \rightarrow \mathbb{R}$. We propose the formation controller as follows

$$
\begin{gathered}
u_{i}=-M \tanh \left(k_{2} \dot{e}_{i X}+k_{1} e_{i X}\right)-M \tanh \left(\rho_{i}\left(e_{i X}, \dot{e}_{i X}\right)\right)+\ddot{\bar{X}}_{i}^{d} \\
\rho_{i}\left(e_{i X}, \dot{e}_{i X}\right)=k_{N} \dot{e}_{i X}
\end{gathered}
$$

where $M$ represents a positive constant scalar. $k_{1}$ and $k_{2}$ are two positive constant gains. The symbol $k_{N}$ represents a positive nonlinear gain. For example, we can select $k_{N}$ as follows

$$
k_{N}=\eta_{1} \exp ^{-\eta_{2} e_{i X}^{2}}
$$

where $\eta_{1} \geq 0, \eta_{2}>0$. The selection of $k_{N}$ in (20) has a physical meaning. When the formation error $e_{i X}$ is large, the effect of $\rho_{i}\left(e_{i X}, \dot{e}_{i X}\right)$ is small. In contrary, when $e_{i X}$ approaches zero, the nonlinear gain $k_{N}$ increases, which can reduce the approaching velocity, such that the overshoot is small.

Proposition 1: The formation controller $u_{i}$ (19) is bounded.
Proof: According to (16), the desired trajectory $\bar{X}_{i}^{d}$ for $\mathrm{UAV} i$ satisfies

$$
\bar{X}_{i}^{d}=\left\{\begin{array}{cc}
\frac{1}{\left|\mathcal{N}_{i}\right|} \sum_{j \in \mathcal{N}_{i}}\left(X_{j}+d_{i j}\right) & \text { if } i \in \mathcal{V}-\mathcal{V}_{L} \\
\frac{1}{\left|\mathcal{N}_{i}+1\right|}\left(\sum_{j \in \mathcal{N}_{i}}\left(X_{j}+d_{i j}\right)+r_{X}+d_{i 0}\right) & \text { if } i \in \mathcal{V}_{L}
\end{array}\right.
$$

where $d_{i j}, d_{i 0} \in \mathbb{R}$ (recall that the subscript " $X$ " has been omitted here) represent some constant offset scalar. Then,

$$
\dot{\bar{X}}_{i}^{d}=\left\{\begin{array}{cc}
\frac{1}{\left|\mathcal{N}_{i}\right|} \sum_{j \in \mathcal{N}_{i}} \dot{X}_{j} & \text { if } i \in \mathcal{V}-\mathcal{V}_{L} \\
\frac{1}{\left|\mathcal{N}_{i}+1\right|}\left(\sum_{j \in \mathcal{N}_{i}} \dot{X}_{j}+\dot{r}_{X}\right) & \text { if } i \in \mathcal{V}_{L}
\end{array}\right.
$$

Let us denote by $\bar{u}_{i}=k_{2} \dot{e}_{i X}+k_{1} e_{i X}$. Then, if $i \in \mathcal{V}-\mathcal{V}_{L}$,

$$
\ddot{\bar{X}}_{i}^{d}=\frac{1}{\left|\mathcal{N}_{i}\right|} \sum_{j \in \mathcal{N}_{i}}\left(-M \tanh \bar{u}_{j}-M \tanh \rho_{j}+\ddot{\bar{X}}_{j}^{d}\right)
$$

such that

$$
\ddot{\bar{X}}_{i}^{d}-\frac{1}{\left|\mathcal{N}_{i}\right|} \sum_{j \in \mathcal{N}_{i}} \ddot{X}_{j}^{d}=-\frac{1}{\left|\mathcal{N}_{i}\right|} \sum_{j \in \mathcal{N}_{i}}\left(M \tanh \bar{u}_{j}+M \tanh \rho_{j}\right)
$$

if $i \in \mathcal{V}_{L}$,

$$
\ddot{\bar{X}}_{i}^{d}=\frac{1}{\left|\mathcal{N}_{i}+1\right|}\left(\sum_{j \in \mathcal{N}_{i}}\left(-M \tanh \bar{u}_{j}-M \tanh \rho_{j}+\ddot{\bar{X}}_{j}^{d}\right)+\ddot{r}_{X}\right)
$$

such that

$$
\begin{aligned}
\ddot{X}_{i}^{d} & -\frac{1}{\left|\mathcal{N}_{i}+1\right|} \sum_{j \in \mathcal{N}_{i}} \ddot{X}_{j}^{d} \\
& =-\frac{1}{\left|\mathcal{N}_{i}+1\right|}\left(\sum_{j \in \mathcal{N}_{i}}\left(M \tanh \bar{u}_{j}+M \tanh \rho_{j}\right)-\ddot{r}_{X}\right)
\end{aligned}
$$

Without loss of generality, we assume that UAVs $1 \sim i$ are leaders, while $i+1 \sim n$ are followers. Then, we rewrite the foregoing equations for all the quadrotors in matrix form as follows

$$
\left[\begin{array}{c}
\ddot{\bar{X}}_{1}^{d} \\
\vdots \\
\ddot{\bar{X}_{n}^{d}}
\end{array}\right]=G^{-1} \cdot\left[\begin{array}{c}
-\frac{1}{\left|\mathcal{N}_{1}+1\right|}\left(\sum_{j \in \mathcal{N}_{1}}\left(M \tanh \bar{u}_{j}+M \tanh \rho_{j}\right)-\ddot{r}_{X}\right) \\
\vdots \\
-\frac{1}{\left|\mathcal{N}_{i}+1\right|}\left(\sum_{j \in \mathcal{N}_{i}}\left(M \tanh \bar{u}_{j}+M \tanh \rho_{j}\right)-\ddot{r}_{X}\right) \\
-\frac{1}{\left|\mathcal{N}_{i+1}\right|} \sum_{j \in \mathcal{N}_{i+1}}\left(M \tanh \bar{u}_{j}+M \tanh \rho_{j}\right) \\
\vdots \\
-\frac{1}{\left|\mathcal{N}_{n}\right|} \sum_{j \in \mathcal{N}_{n}}\left(M \tanh \bar{u}_{j}+M \tanh \rho_{j}\right)
\end{array}\right]
$$

The RFT is usually selected such that its derivatives are bounded. Then, we observe that $\ddot{\bar{X}}_{i}^{d}, i \in \mathcal{V}$ are bounded, since the function $\tanh (\cdot)$ is bounded by 1 . Therefore, we conclude that $u_{i}, i \in \mathcal{V}$ are bounded.

Substituting (19) into the first equation in (18), we obtain

$$
\ddot{e}_{i X}=-M \tanh \bar{u}_{i}-M \tanh \rho_{i}
$$

Proposition 2: The origin of (22) is globally asymptotically stable, if i) $k_{N}$ is given by (20); ii) the gains in $\bar{u}_{i}$ satisfy $k_{1} \geq \eta_{1} k_{2} M$. 
Proof: Let us define a continuously differentiable, radially unbounded positive-definite function as follows

$$
V_{i}=\ln \cosh \left(k_{2} \dot{e}_{i X}+k_{1} e_{i X}\right)+\frac{k_{1}}{2 M} \dot{e}_{i X}^{2}
$$

$V_{i}=0$, if and only if $e_{i X}=0$ and $\dot{e}_{i X}=0$.

Then, the derivative of $V_{i}$ yields

$$
\begin{aligned}
\dot{V}_{i}= & \tanh \bar{u}_{i} \cdot \dot{\bar{u}}_{i}+\frac{k_{1}}{M} \dot{e}_{i X} \ddot{e}_{i X} \\
= & \tanh \bar{u}_{i}\left(k_{2} \ddot{e}_{i X}+k_{1} \dot{e}_{i X}\right)+\frac{k_{1}}{M} \dot{e}_{i X}\left(-M \tanh \bar{u}_{i}-M \tanh \rho_{i}\right) \\
= & \tanh \bar{u}_{i}\left(k_{2}\left(-M \tanh \bar{u}_{i}-M \tanh \rho_{i}\right)+k_{1} \dot{e}_{i X}\right) \\
& -k_{1} \dot{e}_{i X}\left(\tanh \bar{u}_{i}+\tanh \rho_{i}\right) \\
= & -k_{2} M \tanh ^{2} \bar{u}_{i}-k_{2} M \tanh \bar{u}_{i} \tanh \rho_{i}-k_{1} \dot{e}_{i X} \tanh \rho_{i}
\end{aligned}
$$

Then, we have

$$
\dot{V}_{i} \leq-k_{2} M \tanh ^{2}\left(\bar{u}_{i}\right)-k_{1} \dot{e}_{i X} \tanh \rho_{i}+k_{2} M\left|\tanh \bar{u}_{i}\right|\left|\tanh \rho_{i}\right|
$$

According to (19), $\rho_{i}$ has the same sign as $\dot{e}_{i X}$. Then, $-k_{1} \dot{e}_{i X} \tanh \rho_{i} \leq 0$.

- Case 1: If $\left|\rho_{i}\right| \leq\left|\bar{u}_{i}\right|, \quad\left|\tanh \rho_{i}\right| \leq\left|\tanh \bar{u}_{i}\right|$. Then, $\left|\tanh \rho_{i}\right|\left|\tanh \bar{u}_{i}\right| \leq \tanh ^{2} \bar{u}_{i}$. Therefore,

$$
\dot{V}_{i} \leq-k_{1} \dot{e}_{i X} \tanh \rho_{i} \leq 0
$$

We obtain that $\dot{V}_{i}$ is semi-definite negative. We then invoke here the LaSalle's invariance principle. Let us compute the largest invariant set where $\dot{V}_{i}=0$, such that equation (24) equal to zero. We consider the following cases.

- Case a: if $\dot{e}_{i X} \neq 0$, then, according to (25), $\dot{V}_{i}<0$, which contradicts $\dot{V}_{i}=0$.

- Case b: if $\dot{e}_{i X}=0$, then, according to (24), $\dot{V}_{i}=$ $-k_{2} M \tanh ^{2}\left(k_{1} e_{i X}\right)=0$, which implies $e_{i X}=0$.

Therefore, in Case 1, the largest invariant set contains only the origin.

- Case 2: If $\left|\rho_{i}\right|>\left|\bar{u}_{i}\right|,\left|\rho_{i}\right|>\left|\tanh \bar{u}_{i}\right| \geq 0$. Since $k_{1} \geq$ $\eta_{1} k_{2} M$, then, we have $k_{2} M\left|\tanh \bar{u}_{i}\right|<k_{1}\left|\dot{e}_{i X}\right|$. Thus,

$$
\dot{V}_{i} \leq-k_{2} M \tanh ^{2} \bar{u}_{i} \leq 0
$$

We obtain that $\dot{V}_{i}$ is semi-definite negative. As mentioned before, we compute the largest invariant set where $\dot{V}_{i}=0$, such that equation (24) equal to zero. We consider the following cases.

- Case a: if $\left|\bar{u}_{i}\right| \neq 0$, then, according to (26), $\dot{V}_{i}<0$, which contradicts $\dot{V}_{i}=0$.

- Case b: if $\left|\bar{u}_{i}\right|=0$, then, according to (24), $\dot{V}_{i}=$ $-k_{1} \dot{e}_{i X} \tanh \rho_{i}=0$, which implies $\dot{e}_{i X}=0$. Since $\bar{u}_{i}=0$, we obtain that $e_{i X}=0$.

Therefore, in Case 2, the largest invariant set contains only the origin.

Since the largest invariant set contains only the origin, then, according to the LaSalle theorem, the origin of the system (22) is globally asymptotically stable.
Remark 1: The decoupled property of $X$ and $Y$ dynamics permits us to design the formation controller separately, the design procedure of $u_{i}^{Y}$ is the same as $u_{i}^{X}$, such that

$$
u_{i}^{Y}=-M \tanh \left(k_{2} \dot{e}_{i Y}+k_{1} e_{i Y}\right)-M \tanh \rho_{i}\left(e_{i Y}, \dot{e}_{i Y}\right)+\ddot{\bar{Y}}_{i}^{d}
$$

Remark 2: The desired attitude angles for each UAV are obtained by substituting (19) and (27) into (13). Owing to the use of the hyperbolic tangent function, $u_{i}^{X}$ and $u_{i}^{Y}$ are highorder differentiable, such that the derivatives $\dot{\theta}_{i}^{d}, \ddot{\theta}_{i}^{d}$ and $\dot{\phi}_{i}^{d}$, $\ddot{\phi}_{i}^{d}$ are bounded.

Let us reconsider the terms $\delta_{i}^{X}$ and $\delta_{i}^{Y}$ in (14) caused by the tracking error of the attitude angles. We introduce our main result in the following theorem.

Theorem 1: Let $G$ be the interaction matrix of an L-F formation of quadrotors with constant topology. The formation controllers are given in (19) and (27). The attitude is controlled by (3). The altitude is controlled by (9) where $u_{i}^{Z}$ is given in (11). Then, $e_{i X}$ and $e_{i Y}$ converge to zero asymptotically, if i) $G$ is invertible, ii) the initial velocity of each UAV is finite.

Proof: The terms $\delta_{i X}$ and $\delta_{i Y}$ are in terms of the tracking errors of the attitude angles $\left(\Delta \phi_{i}, \Delta \theta_{i}\right.$ and $\left.\Delta \psi_{i}\right)$ and $u_{i}^{Z}$. According to (21), (19), (27) and (11), the controllers $u_{i}^{X}, u_{i}^{Y}$ and $u_{i}^{Z}$ are bounded. Then, according to (13), we know that $\theta_{i}^{d}, \phi_{i}^{d}$ and $\psi_{i}^{d}$ are bounded. The attitude angles are controlled by (3), then, $\Delta \phi_{i}, \Delta \theta_{i}$ and $\Delta \psi_{i}$ are bounded. Therefore, $\delta_{i X}$ and $\delta_{i Y}$ are bounded.

We take $e_{i X}$ for example. Then, (22) becomes

$$
\ddot{e}_{i X}=-M \tanh \bar{u}_{i}-M \tanh \rho_{i}+\delta_{i X}
$$

The derivative of the positive semidefinite function $V_{i}$ in (23) yields

$$
\begin{aligned}
\dot{V}_{i}= & \tanh \bar{u}_{i} \cdot \dot{\bar{u}}_{i}+\frac{k_{1}}{M} \dot{e}_{i X} \ddot{e}_{i X} \\
= & \tanh \bar{u}_{i}\left(k_{2} \ddot{e}_{i X}+k_{1} \dot{e}_{i X}\right)-\frac{k_{1}}{M} \dot{e}_{i X}\left(M \tanh \bar{u}_{i}+M \tanh \rho_{i}-\delta_{i X}\right) \\
= & \tanh \bar{u}_{i}\left(k_{2}\left(-M \tanh \bar{u}_{i}-M \tanh \rho_{i}+\delta_{i X}\right)+k_{1} \dot{e}_{i X}\right) \\
& -k_{1} \dot{e}_{i X}\left(\tanh \bar{u}_{i}+\tanh \rho_{i}+\frac{\delta_{i X}}{M}\right) \\
= & -k_{2} M \tanh ^{2} \bar{u}_{i}-k_{2} M \tanh \bar{u}_{i} \tanh \rho_{i}-k_{1} \dot{e}_{i X} \tanh \rho_{i} \\
& +k_{2} \tanh \bar{u}_{i} \delta_{i X}-\frac{k_{1}}{M} \dot{e}_{i X} \delta_{i X} \\
\leq & -k_{2} M \tanh ^{2} \bar{u}_{i}-k_{2} M \tanh \bar{u}_{i} \tanh \rho_{i}-k_{1} \dot{e}_{i X} \tanh \rho_{i} \\
& +\left(k_{2}\left|\tanh \bar{u}_{i}\right|+\frac{k_{1}}{M}\left|\dot{e}_{i X}\right|\right)\left|\delta_{i X}\right|
\end{aligned}
$$

Since the states of a linear system will not diverge to infinite within finite time interval with bounded control input, then, if the initial condition is in the compact set $\left\{\left(e_{i X}\left(t_{0}\right), \dot{e}_{i X}\left(t_{0}\right)\right) \mid \dot{e}_{i X}\left(t_{0}\right)<\infty\right\}$, we obtain that $\dot{e}_{i X}$ is bounded in finite time interval $\left[t_{0}, t_{1}\right)$, where $t_{1}<\infty$. As analyzed in section II-B, the rotational dynamics of a quadrotor is in the fast time scale $\bar{t}$ with controller (4). Then, there exists a finite time $\bar{t}_{a} \ll t_{1}$ such that the attitude errors $e_{\Theta_{i}}$ enter the neighborhood of the origin, which renders $\left|\delta_{i X}\left(\bar{t}_{a}\right)\right| \leq \zeta$, 
where $\zeta$ is a scalar. Then, for $t>\bar{t}_{a}$, we have

$$
\begin{aligned}
& \left(k_{2}\left|\tanh \bar{u}_{i}\right|+\frac{k_{1}}{M}\left|\dot{e}_{i X}\right|\right)\left|\delta_{i X}\right| \leq \\
& \left|k_{2} M \tanh ^{2} \bar{u}_{i}+k_{2} M \tanh \bar{u}_{i} \tanh \rho_{i}+k_{1} \dot{e}_{i X} \tanh \rho_{i}\right|
\end{aligned}
$$

Then, $\dot{V}_{i} \leq 0$, when $t>\bar{t}_{a}$. Therefore, the semi-global asymptotic stability of the origin of system (28) is derived, when $t>\bar{t}_{a}$.

Remark 3: In theorem 1, if the condition (29) is satisfied when $\bar{t}_{a}=t_{0}$, the semi-global stability of the system (28) is obtained.

\section{Simulation AND EXPERIMENTAL RESUltS}

Heudiasyc laboratory has developed a PC-based simulatorexperiment framework for controlling a quadrotor and also a flock of quadrotors. The programs (written in $\mathrm{C}++$ ) running in the UAVs are the same, both in the simulator and in the embedded processors of real UAVs. When we realize the real-time experiment, the PC acts as a ground station, which is responsible for displaying and sending instructions such as taking off and landing. The UAVs are all autonomous.
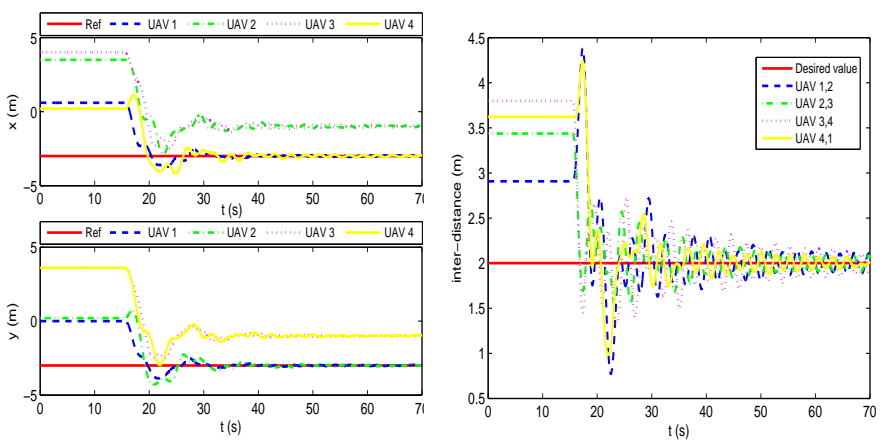

Fig. 2. Formation of four quadrotors without CNF
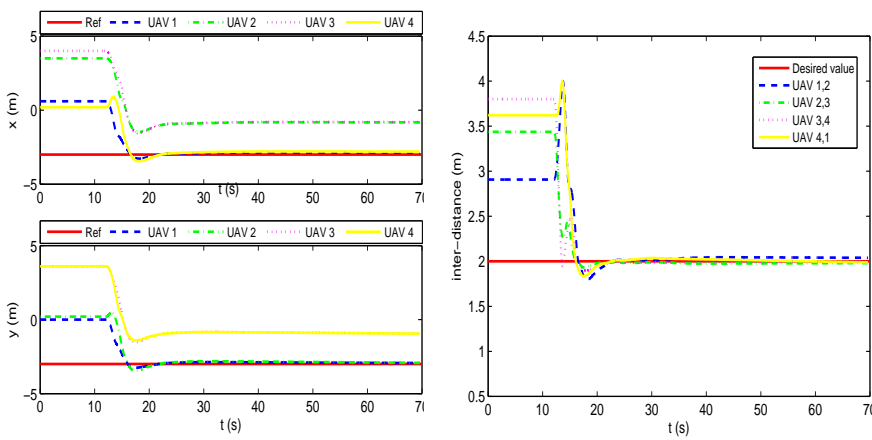

Fig. 3. Formation of four quadrotors with $\mathrm{CNF}$

The formations with and without the CNF are shown in Fig. 2 and Fig.3 respectively. In this test, the formation controllers (19) and (27) are simplified by setting $\ddot{X}_{i}^{d}=$ 0 and $\ddot{Y}_{i}^{d}=0$. We observe that in both formations, the quadrotors are able to converge to the desired positions with hyperbolic tangent function-based controller. However, after adding CNF, the performance is greatly improved.

The foregoing tests validated by using the simulator are carried out in the real-time experiments. The output curves are shown in Fig.4. We observe that the formation with the CNF controller (on the right) has small overshoot and rapid response. The corresponding video is available on the site
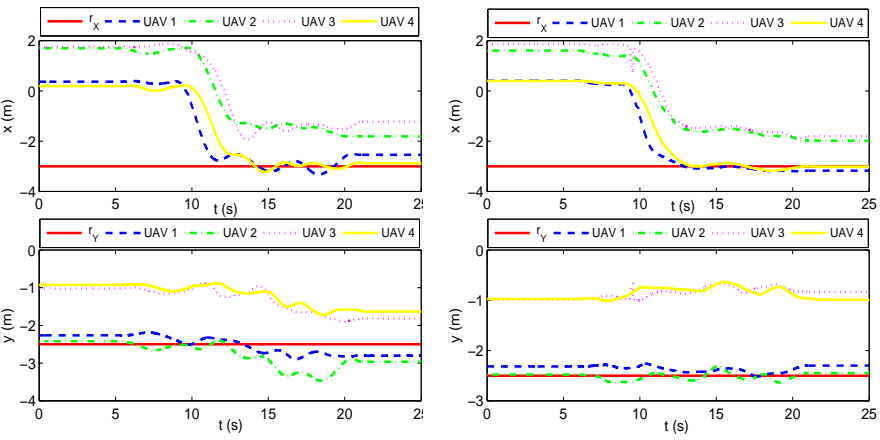

Fig. 4. Real-time formation of four quadrotors. Left: without CNF; Right: with $\mathrm{CNF}$

https://youtu.be/tDBiRAGp6r0

\section{CONCLUSION}

In this paper, the CNF based bounded formation controller is proposed. The hyperbolic tangent function is used to generate a differentiable formation controller. The simulation and the experimental results show that the CNF based formation controller with hyperbolic tangent function have better performance than a controller without $\mathrm{CNF}$ and a controller with a standard saturated function.

\section{REFERENCES}

[1] A. Kushleyev, V. Kumar, and D. Mellinger. Towards a swarm of agile micro quadrotors. In Proceedings of Robotics: Science and Systems, Sydney, Australia, July 2012.

[2] N. Michael, J. Fink, and V. Kumar. Cooperative manipulation and transportation with aerial robots. In Proceedings of Robotics: Science and Systems, Seattle, USA, June 2009.

[3] M. Saska, J. Chudoba, L. Precil, J. Thomas, G. Loianno, A. Tresnak, V. Vonasek, and V. Kumar. Autonomous deployment of swarms of micro-aerial vehicles in cooperative surveillance. In International Conference on Unmanned Aircraft Systems (ICUAS), pages 584-595, May 2014.

[4] P. Dames and V. Kumar. Autonomous localization of an unknown number of targets without data association using teams of mobile sensors. IEEE Transactions on Automation Science and Engineering, $\mathrm{PP}(99): 1-15,2015$.

[5] F. Kendoul, D. Lara, I. Fantoni-Coichot, and R. Lozano. Real-time nonlinear embedded control for an autonomous quadrotor helicopter. AIAA, Journal of Guidance, Control, and Dynamics, 30(4):1049-1061, July-August 2007.

[6] M. Hehn. Quadrocopter trajectory generation and control. In In IFAC World Congress, pages 1485-1491, 2011.

[7] H. Su, M.Z.Q. Chen, J. Lam, and Z. Lin. Semi-global leader-following consensus of linear multi-agent systems with input saturation via low gain feedback. IEEE Transactions on Circuits and Systems I: Regular Papers, 2013.

[8] Z. Lin, M. Pachter, and S. Banda. Toward improvement of tracking performance nonlinear feedback for linear systems. International Journal of Control, 70(1):1-11, 1998.

[9] B.M. Chen, T.H. Lee, Kemao Peng, and V. Venkataramanan. Composite nonlinear feedback control for linear systems with input saturation: theory and an application. IEEE Transactions on Automatic Control, 48(3):427-439, Mar 2003.

[10] Z. Hou and I. Fantoni. Distributed leader-follower formation control for multiple quadrotors with weighted topology. In 10th International Conference on System of Systems Engineering, San Antonio, TX, USA, 2015. 